\title{
Skin 3D Bioprinting. Applications in Cosmetology
}

\author{
Cristina Velasquillo ${ }^{1,2}$, Eduardo A. Galue ${ }^{2}$, Lourdes Rodriquez ${ }^{1}$, Clemente Ibarra $^{1}$, \\ L. Guillermo Ibarra-Ibarra ${ }^{1}$
}

${ }^{1}$ Biotecnología, Instituto Nacional de Rehabilitación, Mexico City, Mexico; ${ }^{2}$ Wake Forest Institute for Regenerative Medicine, Regenerative Medicine, Winston Salem, USA.

Email: mvelasquillo@inr.gob.mx

Received November $16^{\text {th }}, 2012$; revised December $19^{\text {th }}, 2012$; accepted December $28^{\text {th }}, 2012$

\begin{abstract}
Tissue engineering has been used for the treatment of several skin diseases and lesions; however, tissue engineering and regenerative medicine also have a huge potential in cosmetology. They include skin substitutes, cell therapy and wound-healing treatments based on biomaterial-based replacements and 3D systems. This review focuses on the bioprinting technology and how it can improve skin functions, restoring pigmentation or helping hair follicles to develop.
\end{abstract}

Keywords: 3D Bioprinting; Skin Bioprinting; Scaffolds; Human Skin Cells; Cosmetology

\section{Introduction}

Tissue engineering (TE) is a multidisciplinary field that aims at the in vitro engineering of tissues and organs by integrating biology science, materials and biochemical factors.

The skin was the first tissue to have been successfully tissue-engineered in vitro and has also been successfully translated back into clinical applications. This review is focused on the skin-bioprinting technology and how it can be applied in the cosmetic field.

\section{The Skin Structure}

The skin is the largest complex organ in the surface of the human body and it has important functions as temperature regulation, control of evaporation, vitamin D synthesis, maintenance of fluid balance, and protection against pathogens and the environment. It represents about $15 \%$ of the total adult body weight and is composed by three layers: 1) the stratified squamous epithelium, known as "epidermis", includes melanocytes, pilosebaceous follicles and sweat glands, and is originated in the ectoderm; 2) the dermis, separated from the epidermis by the dermal-epidermal junction; and 3 ) the hypodermis [1].

The epidermis is made of various cell types: keratinocytes, representing $90 \%-95 \%$ of the total cell amount; melanocytes, localized in the basal layer of the epidermis and sometimes in the dermis, and non-keratinocytes, including mainly Langerhans and Merkel cells, both representing $5 \%-10 \%$ of the total cell amount. We can also find a cell population known as epidermal stem cells, localized within the basal layer of the interfollicular epidermis and in the bulge of the hair follicle; they have the capacity for self-renewal and repair [2].

Epidermal stem cells within the skin often reside in niches that provide a specialized environment to regulate their proliferation and differentiation. As a result, these cells have the capacity to maintain epidermal homeostasis, proliferating from their basal layer (interfollicular epidermis) through mitotic activity for extended periods of time in the absence of injury or skin damage [3].

This epidermal stem cell population can generate new epithelial cells. With the discovery of this cell population, in the rapidly developing field of epidermal stem cell research, new possibilities have emerged in the design and development of skin tissue regenerative techniques and biomaterials-based skin replacement therapies for the treatment of skin damage due to injuries or aging.

Melanocytes reside in the basal layer of the epidermis along with the proliferative keratinocytes. They produce melanin, a tryptophan-derived lipophilic molecule able to penetrate skin cell membranes. Melanin has been identified in a large number of skin-specific regenerative and anti-apoptotic pathways, including, among others: 1) stimulation of keratinocyte proliferation; 2) stimulation of hair shaft elongation in human hair follicles in vitro at low concentrations, and inhibition at high concentrations; 3) protection against pressure-induced ulcer formation; 4) reduction of thermal skin injury; 5) thermoregulation of skin blood flow; 6) protection against necrosis an lipid peroxidation; and 7) reduction of antioxidative enzymes in skin flaps. They are also associated to 8) a stronger radical scavenger; 9) less ultraviolet-induced DNA frag- 
mentation; and 9) a reduction of caspase 9, 3 and 7 activation, [4]. Melanocytes protect the cells of the integumentary system from a variety of highly damaging environmental factors and are vital to the normal operation of skin.

Adnexa skin structures such as hair and sebaceous glands are vital to the normal functions of the integumentary system. Hair follicles play an important role protecting skin from the outside environment and providing structures within the dermis a communication network with the outer skin. Sebaceous and apocrine glands attach to hair follicles and produce sebum and pheromones, respectively. Hair provides mechanosensory input through bending of the shaft, which allows sensation of slight changes in the environment. Immune cells reside in the follicle, including Langerhans cells, perifollicular macrophages, and mast cells. Follicles also play an important role in wound healing. Cells in the root sheath proliferate in response to an injury and help to regenerate the epidermis. This is important when considering the coverage and repair of large areas for cosmetic purposes to restore normal skin functions [5].

\subsection{Skin TE and Regenerative Medicine}

Tissue engineering is an interdisciplinary field of the regenerative medicine, focused on the creation of tissues and organs with biomaterials and nanostructures to develop, sustain and repair an organ or tissue function. The main goal is to mimic the normal structure of a tissue. Cells and an extracellular matrix (ECM) are used to develop biological substitutes to be assimilated by the body during the tissue regeneration, and cells can also be used in a cell therapy procedure. To achieve these goals, an artificial extra cellular matrix (ECM) and a source of cells are required as well as growth factors (Figure 1).

Tissue engineered replacements play an important role in the treatment of skin wounds, but they are also an option for the treatment of several skin disorders affecting the patients' general appearance and self-esteem; in these cases, skin substitutes and cell therapies may have important clinical applications. These substitutes are created in natural matrices of biodegradable synthetic polymers. The porous structure of these biomaterials provides adequate cell adhesion onto the matrix, and their hydrophilic properties allow transportation of low molecular solutes and nutrients to the cells.

Biomaterials should have some properties such as 1) nontoxic degradation, 2) non-antigenic reaction, and 3) certain physical and mechanical properties, and be able to promote 4) cell proliferation, 5) cell adhesion and 6) cell activity $[6,7]$.

Biological materials with potential for skin tissue regeneration could be "hydrogels" such as collagen, alginate, cellulosa, agarose, chondroitin sulphate, gelatin,

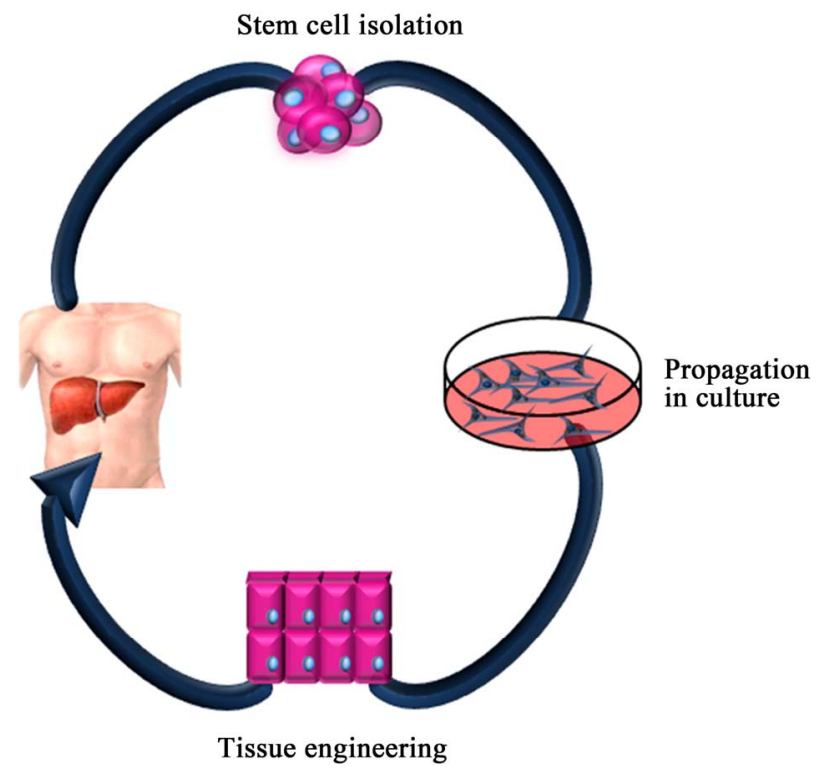

Figure 1. Tissue engineering strategies.

fibrin, hyaluronic acid, collagen type I, type II, type III, chitin, tropoelastin, oxidized cellulose, solubilized alphaelastin and silk fibroin. Their high hydrophilic capacity allows water concentration in the skin [8]. Collagen is also often employed as a cell culture substrate, but it has also been used like the hyaluronic acid (HA) for wound healing.

In addition to biomaterials, some synthetic materials can be used to increase the body's ability for regeneration and self-healing, by means of seeding cells on it to form neo-skin tissue. Synthetic polymers have some advantages, for example: 1) their composition is fully known and understood, 2) they can be tailored to minimize immunological reaction, 3) combine with different copolymers in various proportions, 4) the surface chemistry of these scaffolds allows cell attachment and spreading, 5) they have an appropriate degradation rate in the body, and 6) allow high water content and mechanical properties for cell attachment, cell growth and development of neo-tissues.

Some of the most common synthetic polymers used to make 3-D scaffolds are: PLC (Polycaprolactone), PVA/ PLC (Polycaprolactone-poly-vinil-alcohol), PGA (Polyglicolic Acid), PLLA (Poly-L-Lactide Acid), PLGA (Polyglycolic-polylactide Acid) and polydioxanone [9] (Figure 2).

\subsection{The Bioprinting System Cosmetic Applications}

In recent years, there have been many advances in the three-dimensional (3D) fabrication of biological structures. Researchers are beginning to develop technology that allows design and fabrication, in a controlled manner, 


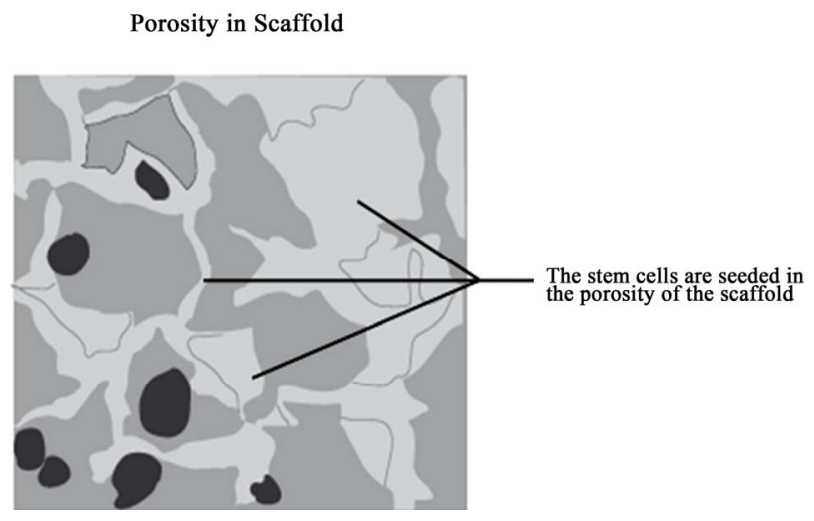

Figure 2. Porosity is important for cells attachment.

of 2D-3D structures composed of biological materials and viable cells. This process is known as "Biofabrication" or "Bioprinting" and faces the challenge to spatially arrange both the living and the non-living components of biological structures to achieve functionality specific to the target application. Development of this technology has been focused on the creation of a $3 \mathrm{D}$ printing machine and the system to monitor and control the fabrication of organs and tissues through an ink-alike fluid called "bio ink" $[10,11]$.

This process has a layer-by-layer additive fabrication approach to create 2D-3D-functional tissue/organ constructs, using cellular spheroids as building blocks. The process is controlled by computer software that designs a 2D-3D pattern through a CAD/CAM system. The hardware consists of a 3-axis motion control stage with an injection syringe for the release of bio ink. The control systems have stepping motors in each axis, motor drivers, and a motion control board in a PC (Figure 3).

As mentioned above, tissues are typically designed in different patterns and biofabricated with different methods, for example, the inkjet printing and the laser-cell patterning printing. The inkjet printing method is based on droplets produced by the application of electrical, thermal and mechanical pulses inside a cartridge with the bio ink to be dispensed. The bio ink is a low-viscosity suspension of biological materials or hydrogels that may or may not contain suspended cells, and the biological structures are created trough a layer-by-layer or drop-bydrop fabrication process.

Typically, modified inkjet printers are used for ejecting small droplets of biological material to the print skin. This method could be applied in cosmetology. The droplets are released layer-by-layer in a $2 \mathrm{D}$ pattern as a simple example for skin tissue. The delivery system of droplets might contain different lines of cells to be used in the skin printing procedure: fibroblast, keratinocytes, melanocytes and sometimes stem cells.

In order to avoid the loss of cells when they are applied to the skin, they can be resuspended with a hydrogel

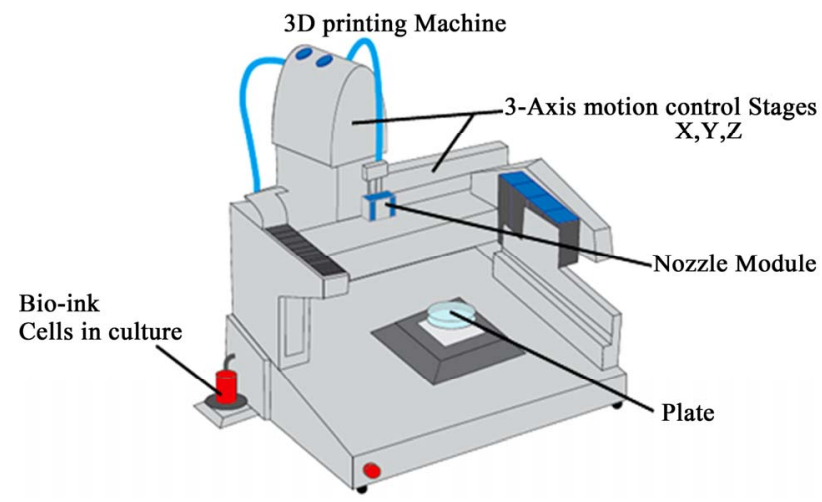

Figure 3. Bioprinting machine.

to form the bio ink that fills the wound surface and promotes skin regeneration [12]. The hydrogel should have some basic properties, like a short gelling time, nonimmunological reactivity, the ability to support cell proliferation and migration, suitable mechanical properties and biodegradability [13].

Using a mixed solution of keratinocytes and fibroblasts, melanocytes, and/or stem cells, the bio-printer may provide a satisfactory model system for treating different structural and cosmetic skin conditions. This technology is currently applied in skin tissue engineering, using a cartridge-based delivery system with a laser scanning system mounted on a portable X-Y-Z plotting system. Data is collected with the laser scanner and pieced to form a wound model of the wound. After this, the delivery system dispenses mixed solutions containing hydrogels and lines of cells to fill the skin surface [14] (Figure 4).

Different skin treatments can be developed, depending on the purpose of the cosmetic application. For example, cultured human melanocytes are increasingly used for treating vitiligo [15]. The bio-printing system could dispense the melanocytes onto the depigmented surface in a personalized and controlled manner, with the advantage that stem cells, growth factors and cytokines can be added at the same time to potentiate treatment effect.

Skin scars are very often hypopigmented due to melanocytes and hair follicle destruction at all levels, and the bioprinting system can control pigmentation by delivering specific amounts of melanocytes and stem cells. It is also possible to induce hair follicle regeneration using stem cells and their niches to develop de novo folliculogenesis via replacement with hair follicle-inductive dermal cells, to induce self-assembly of skin derived epithelial and mesenchymal cells [16,17]. However, it remains uncertain if it is possible to generate a hair follicle by intracutaneous transplantation to provide fully functional hair regeneration, including hair shaft elongation, hair cycles, connections with surrounding tissues, and 


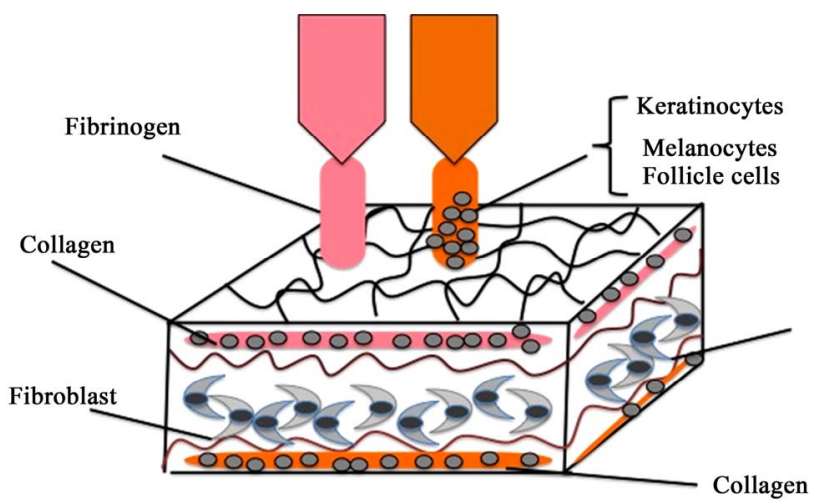

Figure 4. Skin bioprinting for functional skin.

regeneration of stem cells and their niches. At present, functional hair follicle regeneration used for treating androgenic alopecia requires a clinical therapy of autologous follicular stem cells transplantation $[18,19]$.

With the bioprinting system, the follicle-bioengineering uses biomaterials to provide connections between the cell lines to generate skin functionality. This system also could provide various layers constructed block-by-block or various tissues constructed layer-by-layer with specific cells for each skin level (epidermis, dermis, fatty subcutis, hypodermis) to promote integration and formation of the vascular and nerve networks for the complete regeneration of functional skin.

\section{Acknowledgements}

This study was supported by CONACYT Sectoriales with grant 161687 and the INR board for the fellow scholarship at WFIRM.

\section{REFERENCES}

[1] G. Murphy, "Histology of the Skin," In: R. Elenitsas, C. Jaworsky and B. Johnson, Eds., Lever's Histopathology of the Skin, Lippincott-Raven, Philadelphia, 1997, pp. 550.

[2] S. Chunmeng and Ch. Tianmin, "Skin: A Promising Reservoir for Adult Stem Cell Populations," Medical Hypotheses, Vol. 62, No. 5, 2004, pp. 683-688. doi:10.1016/j.mehy.2003.12.022

[3] H. Lin, "The Stem-Cell Niche Theory: Lessons from Flies," Nature Reviews Genetics, Vol. 3, No. 12, 2002, pp. 931940. doi:10.1038/nrg952

[4] T. W. Fischer, A. Slominski, M. A. Zmijewski, R. J. Reiter and R. Paus, "Melatonin as a Major Skin Protectant: From Free Radical Scavenging to DNA Damage Repair," Experimental Dermatology, Vol. 17, No. 9, 2008, pp. 713730. doi:10.1111/j.1600-0625.2008.00767.x

[5] R. Paus and G. Cotsarelis, "The Biology of Hair Follicles," New England Journal of Medicine, Vol. 341, No. 7, 1999, pp. 491-497. doi:10.1056/NEJM199908123410706

[6] W. Liu and Y. Cao, "Application of Scaffold Materials in
Tissue Reconstruction in Immunecompetent Mammals: Our Experience and Future Requirements," Biomaterials, Vol. 28, 2007, pp. 5078-5086. doi:10.1016/j.biomaterials.2007.07.028

[7] E. Sachlos and J. T. Czernuszka, "Making Tissue Engineering Scaffolds Work. Review: The Application of Solid Freeform Fabrication Technology to the Production of Tissue Engineering Scaf-Folds," European Cells and Materials, Vol. 5, 2003, pp. 29-39; discussion 39-40.

[8] B. M. Min, S. W. Lee, J. N. Lim, et al., "Chitin and ChitoSan Nanofibers: Electrospinning of Chitin and Deacetylation of Chitin Nanofibers," Polymer, Vol. 45, No. 21, 2004 , pp. 7137-7142. doi:10.1016/j.polymer.2004.08.048

[9] S. J. Peter, M. J. Miller, A. W. Yasko, M. J. Yaszemski and A. G. Mikos, "Polymer Concepts in Tissue Engineering," Journal of Biomedical Materials Research, Vol. 43, No. 4, 1998, pp. 422-427.

doi:10.1002/(SICI)1097-4636(199824)43:4<422::AID-JB M9>3.0.CO;2-1

[10] A. Mohamed and M. Xing, "Nanomaterials and Nanotechnology for Skin Tissue Engineering," International Journal of Burns and Trauma, Vol. 2, No. 1, 2012, pp. $29-41$.

[11] W. C. Wilson Jr. and T. Boland, "Cell and Organ Printing 1; Protein and Cell Printers," The Anatomical Record: Part A, Vol. 272, No. 2, 2003, pp. 491-496.

[12] J. J. Yoo, A. Atala, K. W. Binder, W. Zhao, D. Dice and T. Xu, Delivery System Office, United States Patent \& Trademark.12/986, 2011.

http://appft1.uspto.gov/netacgi/nphParser?Sect11/4PTO1 \&Sect21/4HITOFF\&d1/4PG01\&p1/41\&u1/4/netahtml/PT O/srchnum.html\&r1/41\&f1/4G\&11/450\&s11/4201101726 11.PGNR

[13] Y. Li, J. Rodrigues and H. Tomas, "Injectable and Biodegradable Hydrogels: Gelation, Biodegradation and Biomedical Applications," Chemical Society Reviews, Vol. 41, No. 6, 2012, pp. 2193-2221. doi:10.1039/c1cs15203c

[14] L. Koch, A. Deiwick, S. Schlie, S. Michael, M. Gruene, V. Coger, D. Zychlinski, A. Schambach, K. Reimers, P. M. Vogt and B. Chichkov, "Skin Tissue Generation by Laser Cell Printing," Biotechnology and Bioengineering, Vol. 109, No. 7, 2012, pp. 1855-1863. doi:10.1002/bit.24455

[15] R. Czajkowski, "BRAF, HRAS, KRAS, NRAS and CDKN2A Genes Analysis in Cultured Melanocytes Used for Vitiligo Treatment," International Journal of Dermatology, Vol. 50, No. 2, 2011, pp.180-183. doi:10.1111/j.1365-4632.2010.04675.x

[16] W. C. Weinberg, et al., "Reconstitution of Hair Follicle Development in Vivo: Determination of Follicle Formation, Hair Growth, and Hair Quality by Dermal Cells," Journal of Investigative Dermatology, Vol. 100, No. 3, 1993, pp. 229-236. doi:10.1111/1523-1747.ep12468971

[17] J. Kishimoto, et al., "Selective Activation of the Versican Promoter by Epithelial Mesenchymal Interactions during Hair Follicle Development," Proceedings of the National Academy of Sciences USA, Vol. 96, No. 13, 1999, pp. 7336-7341. doi:10.1073/pnas.96.13.7336

[18] W. Unger, R. Shapiro, M. A. Unger and U. Unger, "Hair 
Transplantation," 5th Edition, Informa USA, New York, 2010. doi:10.3109/9781616310073

[19] C. M. Chuong, G. Cotsarelis and K. Stenn, "Defining
Hair Follicles in the Age of Stem Cell Bioengineering," Journal of Investigative Dermatology, Vol. 127, No. 9, 2007, pp. 2098-2100. doi:10.1038/sj.jid.5700947 